CONF-950339--1.

LCRL-JC-120092

PREPRINT

\title{
VARIATIONAL STRUCTURE OF INVERSE PROBLEMS IN WAVE PROPAGATION AND VIBRATION
}

\author{
James G. Berryman
}

This paper was prepared for submittal to the Institute of Mathematics and Its Applications Workshop on Inversion Problems in Wave Propagation Minneapolis, Minnesota March 6-17, 1995

March 1995

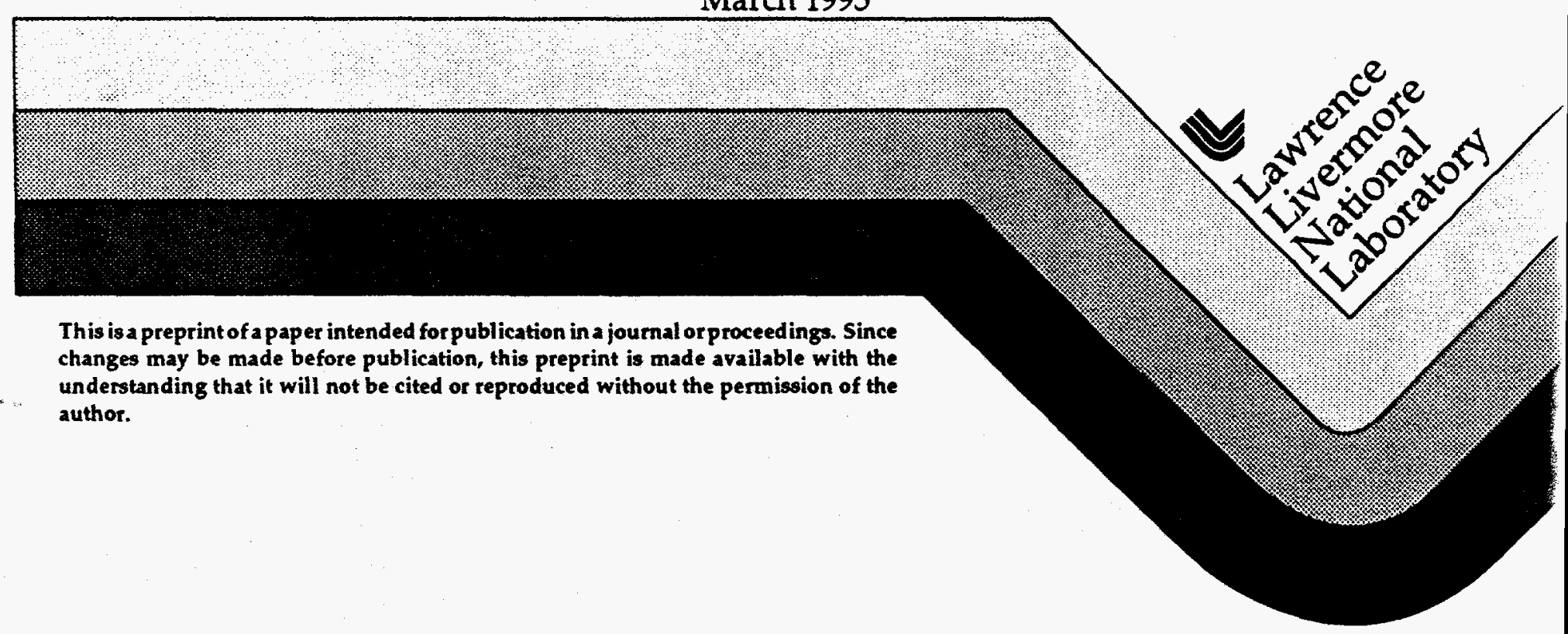




\section{DISCLAIMER}

This report was prepared as an account of work sponsored by an agency of the United States Government. Neither the United States Government nor any agency thereof, nor any of their employees, make any warranty, express or implied, or assumes any legal liability or responsibility for the accuracy, completeness, or usefulness of any information, apparatus, product, or process disclosed, or represents that its use would not infringe privately owned rights. Reference herein to any specific commercial product, process, or service by trade name, trademark, manufacturer, or otherwise does not necessarily constitute or imply its endorsement, recommendation, or favoring by the United States Government or any agency thereof. The views and opinions of authors expressed herein do not necessarily state or reflect those of the United States Government or any agency thereof. 


\section{DISCLAIMER}

Portions of this document may be illegible in electronic image products. Images are produced from the best available original document. 


\title{
Variational Structure of Inverse Problems in Wave Propagation and Vibration
}

\author{
James G. Berryman \\ Lawrence Livermore National Laboratory \\ P. O. Box 808 L-202 \\ Livermore, CA 94551-9900
}

OISTRIBUTION OE IHIS DOCUMENT IS UNLIMITED

yl

Version date: March 16, 1995 


\begin{abstract}
Practical algorithms for solving realistic inverse problems may often be viewed as problems in nonlinear programming with the data serving as constraints. Such problems are most easily analyzed when it is possible to segment the solution space into regions that are feasible (satisfying all the known constraints) and infeasible (violating some of the constraints). Then, if the feasible set is convex or at least compact, the solution to the problem will normally lie on the boundary of the feasible set. A nonlinear program may seek the solution by systematically exploring the boundary while satisfying progressively more constraints. Examples of inverse problems in wave propagation (traveltime tomography) and vibration (modal analysis) will be presented to illustrate how the variational structure of these problems may be used to create nonlinear programs using implicit variational constraints.
\end{abstract}

\title{
1 Introduction
}

Although the most common method used to analyze inverse problems is perturbation theory and/or linearization, it is generally recognized that most inverse problems of practical interest are mathematically nonlinear. The inherent nonlinearities lead to the necessity of distinguishing local minima from global minima. Optimization methods based on objective functionals typically produce "solutions" that minimize the functional "locally" - i.e., in the vicinity of the starting point of the minimization process.

The methods discussed in the present paper bave been developed specifically to improve our insight into the structure of certain classes of nonlinear inverse problems. Furthermore, once this structure is understood, solutions of the inverse problems using nonlinear programming methods are immediately suggested by the particular variational structure observed.

\section{String Vibration Analysis}

The equation of vibration for a simple string is

$$
\rho \frac{\partial^{2} u}{\partial t^{2}}=T \frac{\partial^{2} u}{\partial x^{2}}
$$

where $t$ is the time, $x$ is the spatial coordinate of the string along its length, $\rho(x)$ is the density distribution of the string, $T$ is the tension in the string (assumed constant), and $u(x, t)$ is the normal displacement of the atring from its equilibrium position as a function of both position and time. The ends of the string are fixed, so the boundary conditions are $u(0, t)=0=u(1, t)$. We assume that the string's temporal motion may be decompceed into its Fourier components so that we may study standing waves on the plucked atring. The time dependence is anumed to separate out in one of the forms sin $\omega t$, cos $\omega t$, or exp $i \omega t$, where $\omega=2 \pi f$ is angular frequency with dimensions of radians/sec, while $f$ is frequency in $H z$. Then, the equation (1) reduces to

$$
-\omega^{2} p u=T u_{2 x}
$$

where we have now introduced the subacript notation for derivatives such that $u_{z} \equiv \partial u / \partial x$, $u_{x z} \equiv \partial^{2} u / \partial x^{2}$, etc. 


\section{Modal analysis for string density}

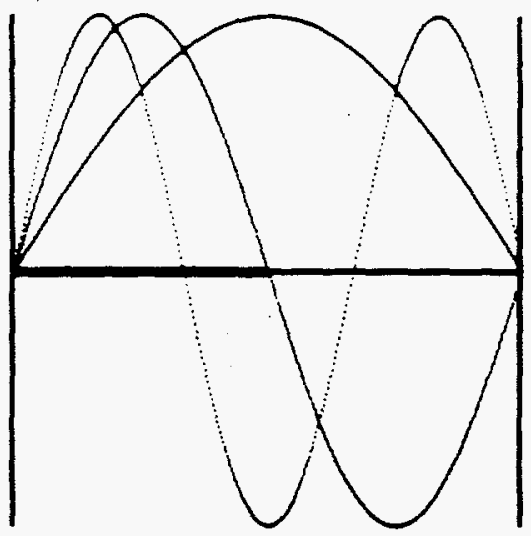

Figure 1: Density distribution of the string may be determined by analyzing the eigenfrequencies associated with its modes of vibration.

We assume that the tension $T$ in the string is known, but the density distribution $\rho(x)$ of the string is unknown. Our goal is to determine to what extent $\rho(x)$ can be determined using knowledge of modes of vibration of the string. In particular, we assume that some measurements of the vibration frequencies for standing waves have been made.

Let $\omega_{n}$ be the eigenfrequency and $u_{n}(x)$ the eigenfunction of the $n$-th Fourier component for $n \geq 1$. Higher $n$ corresponds to eigenfunctions with more internal nodes, e.g., $n=1$ has no internal nodes, $n=2$ has one internal node, etc. Then, after choosing unit tension $T=1$, we multiply (2) by $u_{n}(x)$ and integrate along the length of the string to show that

$$
\omega_{n}^{2}(\rho)=\frac{\int_{0}^{1} u_{n, x}^{2}(x) d x}{\int_{0}^{1} \rho(x) u_{n}^{2}(x) d x} .
$$

To arrive at (3), we integrated once by parts $\left(\int u_{n} u_{n, x=} d x=-\int u_{n, x}^{2} d x\right)$ using the fact that $u_{n}(0)=0=u_{n}(1)$ to eliminate the boundary contribution. Equation (3) is an identity satisfied by the $n$-th eigenfrequency and relating it to the integrated properties of the $n$-th eigenfunction.

Now the Rayleigh-Ritz method for characterizing eigenvalues [Courant and Hilbert, 1953] may be applied to the string problem and it shows that

$$
\omega_{1}^{2}(\rho) \leq R\left[\rho, v_{1}\right] \equiv \frac{\int_{0}^{1} v_{1, z}^{2}(x) d x}{\int_{0}^{1} \rho(x) v_{1}^{2}(x) d x},
$$

where $R$ is the Rayleigh-Ritz quotient with $v_{1}(x)$ being any trial function satisfying the boundary conditions $v_{1}(0)=0=v_{1}(1)$ whose first derivative with respect to $x$ is finite everywhere along the interval containing the string. When the string density distribution $p(x)$ is known, the Rayleigh-Ritz method is often used as a numerical method for finding eatimates of both 


\section{Scaling $\rho$ to find boundary point}

Density model $\rho$

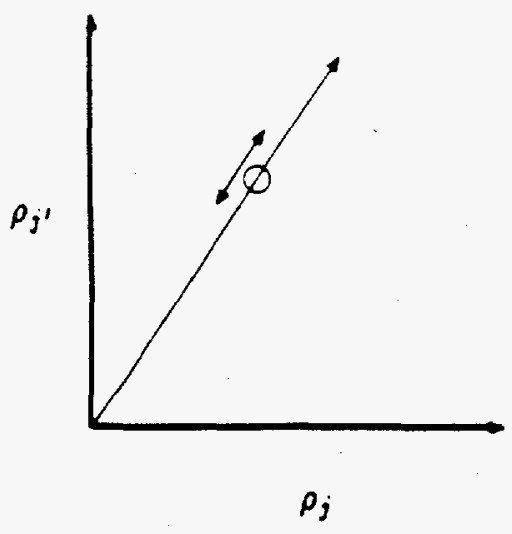

Vibration data $\omega$

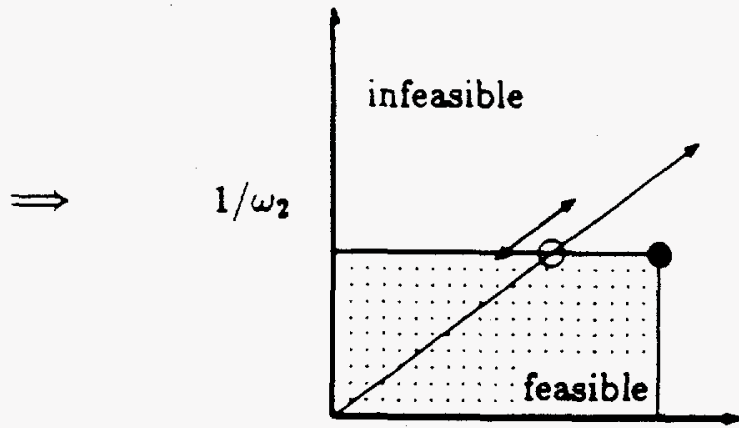

$1 / \omega_{1}$

Figure 2: Feasible part of the density space is determined implicitly by the explicit boundaries defined by the frequency data.

the eigenfrequency $\omega_{1}$ and the eigenfunction $u_{1}(x)$; the theory shows that $R\left[\rho, v_{1}\right] \equiv \omega_{1}^{2}(\rho)$ if and only if $v_{1}(x)=u_{1}(x)$ almost everywhere. Similarly, if we impose constraints on the trial function such as requiring $v_{n}(x)$ to have $n-1$ interior nodes [Coddington and Levinson, 1955], then (4) generalizes to

$$
\omega_{n}^{2}(\rho) \leq R\left[\rho, v_{n}\right] \equiv \frac{\int_{0}^{1} v_{n, x}^{2}(x) d x}{\int_{0}^{1} \rho(x) v_{n}^{2}(x) d x} .
$$

The characteristic frequencies (the ws) are those defined in (3) for the higher order modes. We will sometimes refer to the equations (4) and (5) as the "forward problem," since in these equations we assume that the density distribution is a known quantity. The "inverse problem" is the harder problem of taking measured values of the eigenfrequencies $\omega_{n}$ and attempting to solve for the unknown denaity diatribution $\rho(x)$.

\subsection{Feasibility analysis}

There are two tricks that make it possible in some cases to use the variational functionals to analyze inversion problems. The first trick is a result of what we call the "scale invariance" property of the eigenfunctions. For a given density distribution $p$ and its corresponding eigenfunction $u(x)$, the only effect of multiplying $\rho$ by a constant $\lambda$ is to change the eigenfrequency by the factor $1 / \sqrt{\lambda}$. This result follows since

$$
R[\lambda \rho, u]=R[\rho, u] / \lambda,
$$

which implies that $\omega(\lambda \rho)=\omega(\rho) / \sqrt{\lambda}$. We can conclude that each eigenfunction $u(x)$ is determined only by the relative variations in the density, not by the abeolute scale. 
The second trick is a result of the linear dependence of the denominator of the RayleighRitz functional on the density distribution $\rho$. We can take advantage of this linearity in $\rho$ with greater ease by working with the reciprocal of $R$, so we first note that

$$
\frac{1}{R[\rho, v]}=\frac{\int \rho(x) v^{2}(x) d x}{\int v_{x}^{2}(x) d x} \leq \omega^{-2}(\rho) .
$$

Now, if we have made measurements of some of the eigenfrequencies of the string, we can ask the following question: Are there density distributions that violate the inequality in $(7)$ ? That is, if we try to compute the left hand side of (7) using an arbitrary trial density distribution $\tau(x)$, when will the inequality be satisfied and when will it be violated? We use the answers to these questions to define two distinct classes of trial model density distributions $\tau(x)$ :

$$
\text { If } \frac{1}{R\left[\tau, v_{n}\right]} \leq \omega_{n}^{2}(\rho) \text { for each measured frequency } \omega_{n} \text {, then } r(x) \text { is fecrible. }
$$

However,

$$
\text { if } \frac{1}{R\left[r, v_{n}\right]}>\omega_{n}^{2}(\rho) \text { for any measured frequency } \omega_{n} \text {, then } r(x) \text { is infeasible. }
$$

As defined here, the concepts of feasible and infeasible trial density distributions depend explicitly on the available data $\omega_{n}$ and implicitly on the trial functions $v_{n}(x)$. However, it is also possible to show [Berryman, 1991] that universal (global) feasible and infeasible sets exist that are independent of the particular trial functions chosen (dependence on the particular choice of those measured frequencies which are used in the analysis still remains). The concepts of feasible and infeasible sets are commonly found in texts on nonlinear programming methods [Fiacco and McCormick, 1990]. Since the method we develop for solving the inverse problem is a type of nonlinear programming method, it is not surprising that these concepts also arise in the context of numerical methods for solving inverse problems.

Now, we derive a very useful property of the class of feasible density distributions. Consider two feasible trial densities $\tau_{0}(x)$ and $\tau_{b}(x)$, which therefore (by definition) satisfy

$$
\frac{1}{R\left[\tau_{a}, v\right]} \leq \omega^{-2}(\rho) \text { and } \frac{1}{R\left[r_{b}, v\right]} \leq \omega^{-2}(\rho) \text {. }
$$

Let $\lambda$ be a number in the range $0 \leq \lambda \leq 1$. Then, taking a linear combination of the two expressions in (10) givea

$$
\frac{\lambda}{R\left[r_{a}, v\right]}+\frac{1-\lambda}{R\left[r_{b}, v\right]} \leq \omega^{-2}(\rho)
$$

But, the left hand side of (11) may be rewritten as

$$
\frac{\int\left[\lambda r_{a}(x)+(1-\lambda) r_{b}(x)\right] v^{2}(x) d x}{\int v_{x}^{2}(x) d x}=\frac{1}{R\left[r_{\lambda}, v\right]},
$$

where we have defined the convex combination of the trial densities to be

$$
r_{\lambda}(x) \equiv \lambda r_{a}(x)+(1-\lambda) r_{b}(x) \text {. }
$$


Combining (11) and (12) shows that

$$
\frac{1}{\left.2 \tau_{\lambda}, v\right]} \leq \omega^{-2}(\rho)
$$

But any trial function that satisfies (14) for all measured frequencies is by definition feasible. So, if $r_{a}(x)$ and $r_{b}(x)$ are both feasible, their convex combination $r_{\lambda}(x)$ is also feasible.

The definition of a convex set is this: a set such that the convex combination - i.e., $r_{\lambda}=\lambda r_{a}+(1-\lambda) r_{b}$ - of any two members is also a member of the set. So, this fact implies that the set of all feasible density distributions for a given set of vibration data is a convex set. In general, convex sets are very useful in computations because they are often compact and always have smooth boundaries.

Now, the first trick (scale invariance) plays an interesting and important role in the analysis. Suppose we have any trial density distribution $\tau_{w}$ and we have found the eigenfunctions $w_{n}(x)$ and eigenvalues $\omega_{n}\left(\tau_{w}\right)$ associated with this distribution. Then, the eigenfunctions $w_{n}(x)$ are also the eigenfunctions for all densities of the form $\gamma r_{w}(x)$, where $\gamma$ is an arbitrary positive scalar. It is not hard to show [Berryman, 1991] that there always exists a choice of $\gamma=\gamma_{w}$ such that $\gamma_{w} \tau_{w}(x)$ lies exactly on the boundary of the convex feasible set for the inversion problem. It follows then that; if $\gamma \leq \gamma_{w}$, the density $\gamma \tau_{w}$ lies in the feasible part of the model space; while, if $\gamma>\gamma_{w}$, the density $\gamma \tau_{w}$ lies in the infeasible part. This characteristic of the feasible set allows us to produce a simple geometrical interpretation of the feasible set as we will show.

In particular, for the vibrating string inversion problem, the feasible set of model densities is compact and occupies a convex region in the neighborhood of the origin of the model space [i.e., near $\rho(x) \equiv 0$ ]. We can easily prove this statement. First note a physical density must be nonnegative, so the general model space is the convex set of all possible nonnegative density distributions. Then, by taking the trial eigenfunction to be $v_{1}(x)=\sin \pi x$ with the trial density $r(x)=r_{0}($ constant $)$, and noting that

$$
\frac{1}{R\left[\tau_{0}, v_{1}\right]}=\frac{\tau_{0} \int \sin ^{2} \pi x d x}{\pi^{2} \int \cos ^{2} \pi x d x}=\frac{\tau_{0}}{\pi^{2}}
$$

satisfies the feasibility constraint if

$$
\tau_{0} \leq \pi^{2} \omega_{1}^{-2}(\rho)
$$

The first measured eigenfrequency $\omega_{1}$ then determines an upper bound on the value of $r_{0}$ for constant density models. For nonconstant models such as the two component string with $r(x)=\tau_{a}$ for $0 \leq x<\frac{1}{2}$ and $r(x)=\tau_{b}$ for $\frac{1}{2}<x \leq 1$, the same argument shows that

$$
\frac{1}{R\left[r, v_{1}\right]}=\frac{r_{a} \int_{0}^{\frac{1}{2}} \sin ^{2} \pi x d x+r_{b} \int_{\frac{1}{2}}^{1} \sin ^{2} \pi x d x}{\pi^{2} \int_{0}^{1} \cos ^{2} \pi x d x}=\frac{r_{0}+r_{b}}{2 \pi^{2}} \leq \omega_{1}^{-2}(\rho) .
$$

Thus, (17) show that $\pi$ is bounded above by the straight line

$$
r_{b} \leq 2 \pi^{2} \omega^{-2}(\rho)-r_{a} \text {, }
$$

producing tighter bounds on $r_{b} r_{a}$ increases. When $r_{a}=r$, this problem reduces to the constant model result found earlier.

These simple examples can be used to produce further restrictions on model densities, as was shown in the talk. 


\section{Mapping the feasibility boundary}

Density mode! $\rho$

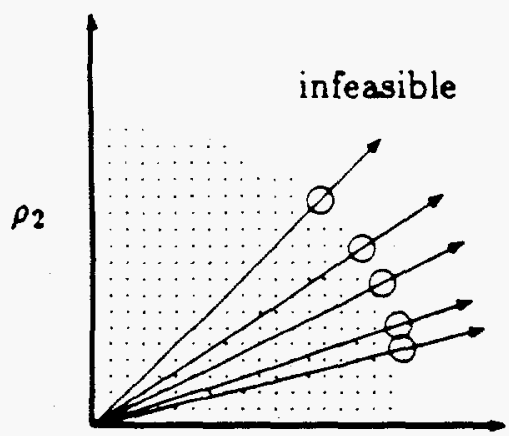

$P_{1}$
Vibration data w

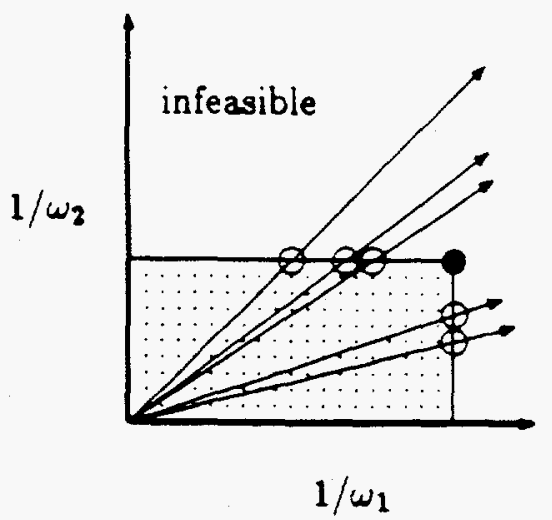

Figure 3: By scaling many density distributions, the location of the feasibility boundary can be mapped.

\subsection{Algorithm}

Now we can describe a general algorithm for solving the inverse problem for a two-segment string. This aigorithm involves a lot of forward modeling, but will always produce a good approximation to the solution. First, note that the symmetry of the problem guarantees that if $\left(\tau_{1}, \tau_{2}\right)$ solves the problem then so does $\left(\tau_{2}, \tau_{1}\right)$. (We are presently considering only the frequencies as dats. This nonuniqueness of the solution can be easily removed by considering the eigenfunctions as data as well.) Thus, we only need to consider half of the positive quadrant, say for models such that $r_{1} \geq r_{2}$. Then, picking modele evenly spaced in angle for (say) 10 angles up to $\theta=\pi / 4, s 0\left(\tau_{1}, \tau_{2}\right)=\left(\tau_{0} \cos \theta, \tau_{0} \sin \theta\right)$ with $\theta=\pi / 40, \pi / 20, \ldots, \pi / 4$. (The precise value of $\tau_{0}$ does not matter, since these points will eventually be scaled onto the feasibility boundary.) Having chosen a set of initial points, we now do forward modeling on each of these string models. After scaling to the feasibility boundary, we check satisfaction of the data and pick the two adjacent points that best satisfy the data. Then, we divide the angular region bracketed by these two angles into 10 smaller angles, and repeat this process until we have a satisfactory solution. The algorithm just described is basically a "shooting" algorithm for the inverse problem.

For more complex modela involving three or more string segments, the inversion algorithm is complicated only by the need to define an efficient means of choosing points in the model space for each forward modeling phase of the calculation. It is also clear that algorithms of this type can easily be parallelized, with an individual processor assigned to do a single forward modeling computation at each step of the algorithm. 


\section{Traveltime Inversion Problem}

A typical problem arising in seismic traveltime inversion in 2- and 3-dimensional heterogeneous media is the need to infer the (isotropic) compressional-wave slowness (reciprocal of velocity) distribution of a medium, given a set of observed first-arrival traveltimes between sources and receivers of known location within the medium [Dines and Lytle, 1979; Lytle and Dines, 1980). This problem is common for crosswell seismic transmission tomography imaging a 2-D region occupying the plane between two vertical boreholes in oil field applications. We could also consider the problem of inverting for wave slowness when the absolute traveltimes are not known perfectly, as is normally the case in earthquake seismology for whole Earth structure Aki and Richards, 1980].

\subsection{Wave slowness models}

When a sound wave or seismic wave is launched into a medium, it takes time for the influence of the wave to progress from a point close to the source to a more distant point. The time taken by the wave to travel from one point of interest to the next is called the traveltime. The local slowness is the inverse of the local wave speed. It is most convenient to develop inversion and tomography formulas in terms of wave slowness models, because the pertinent equations are linear in slowness.

We might consider three kinds of slowness models. We could allow the slowness to be a general function $s(x)$ of the position $x$. However, we often make one of two more restrictive assumptions that (i) the model comprises homogeneous cells (in 2-D), or blocks (in 3-D), with $s_{j}$ then denoting the slowness value of the $j$ th cell, or block. Or (ii) the model is defined in terms of a grid with values of slowness assigned at the grid points together with some interpolation scheme (bilinear, trilinear, spline, etc.) to specify the values between grid points. Of course, as cells/blocks become smaller and smaller (down to infinitesimal), we can think of cells/blocks of constant slowness as a special case of continuous models, or of continuous models as a limiting case of cells/blocks.

When it is not important which type of slowness model is involved, we refer to the model abstractly as a vector $s$ in a vector space $S$. For a block model with $n$ blocks, we have $S=\mathbf{R}^{n}$, the n-dimensional Euclidean vector space. A continuous slowness model, on the other hand, is an element of a function space, e.g., the set of continuous functions of three real variables. No matter how we parameterize the model, our models necessarily have far fewer parameters than the actual medium they are intended to represent. Thus, our models are analogous to cartoons, trying to capture the main features with the minimum of detail.

\subsection{Fermat's principle and traveltime functionals}

The traveltime of a seismic wave is the integral of slowness along a ray path connecting the source and receiver. To make this more precise, we will define two types of functionals for traveltime.

Let $P$ denote an arbitrary path connecting a given source and receiver in 1 slowneas model 8. We will refer to $P$ as a trial ray path. We define a functional $r^{P}$, which yields the traveltime 
along path $P$. Letting $\mathbf{s}$ be the continuous slowness distribution $s(\mathbf{x})$, we have

$$
T^{P} \mathbf{s}^{i}=\int_{P} s(\mathbf{x}) d d^{P}
$$

where $d l^{P}$ denotes the infinitesimal distance along the path $P$.

Fermat's principle states that the correct ray path between two points is the one of least overall traveltime, i.e., it minimizes $r^{P}(\mathbf{s})$ with respect to path $P$.

Let us define a second traveltime functional $\tau^{*}$ to be the functional that yields the traveltime along the Fermat (least-time) ray path. Fermat's principle then states

$$
r^{*}[\mathbf{s}]=\min _{P \in P a t h s} r^{P}[\mathbf{s}]
$$

where Paths denotes the set of all continuous paths connecting the given source and receiver. The particular path that produces the minimum in $(20)$ is denoted $P^{*}$. If more than one patb produces the same minimum traveltime value, then $P^{*}$ denotes any particular member in this set of minimizing paths.

Substituting (19) into (20), we have Fermat's principle of least time:

$$
r^{*}[\mathbf{s}]=\int_{P^{*}} s(\mathbf{x}) d l^{P^{*}}=\min _{P} \int_{P} s(\mathbf{x}) d l^{P}
$$

The traveltime functional $r^{*}[8]$ is well-known to be stationary with respect to small variations in the path $P^{*}(s)$.

\subsection{Seismic inversion and tomography}

Suppose we have a set of observed traveltimes, $t_{1}, \ldots, t_{m}$, from $m$ source-receiver pairs in a medium of slowness $s(x)$. Let $P_{i}$ be the Fermat ray path connecting the $i$ th source-receiver pair. Neglecting observational errors, we can write

$$
\int_{P_{i}} s(\mathbf{x}) d d^{P_{i}}=t_{i}, \quad i=1, \ldots, m .
$$

Given a block slownese model, let $l_{i j}$ be the length of the ith ray path through the $j$ th cell:

$$
l_{i j}=\int_{P_{i} \cap \text { cell }_{j}} d{ }^{P_{i}} .
$$

For $n$ cells, Eq. (22) can then be written

$$
\sum_{j=1}^{n} l_{i j} s_{j}=t_{i}, \quad i=1, \ldots, m .
$$

Note that for any given $i$, the ray-path lengths $l_{i j}$ are zero for most cells $j$, as a given ray path will in general intersect only a few of the cells in the model. 


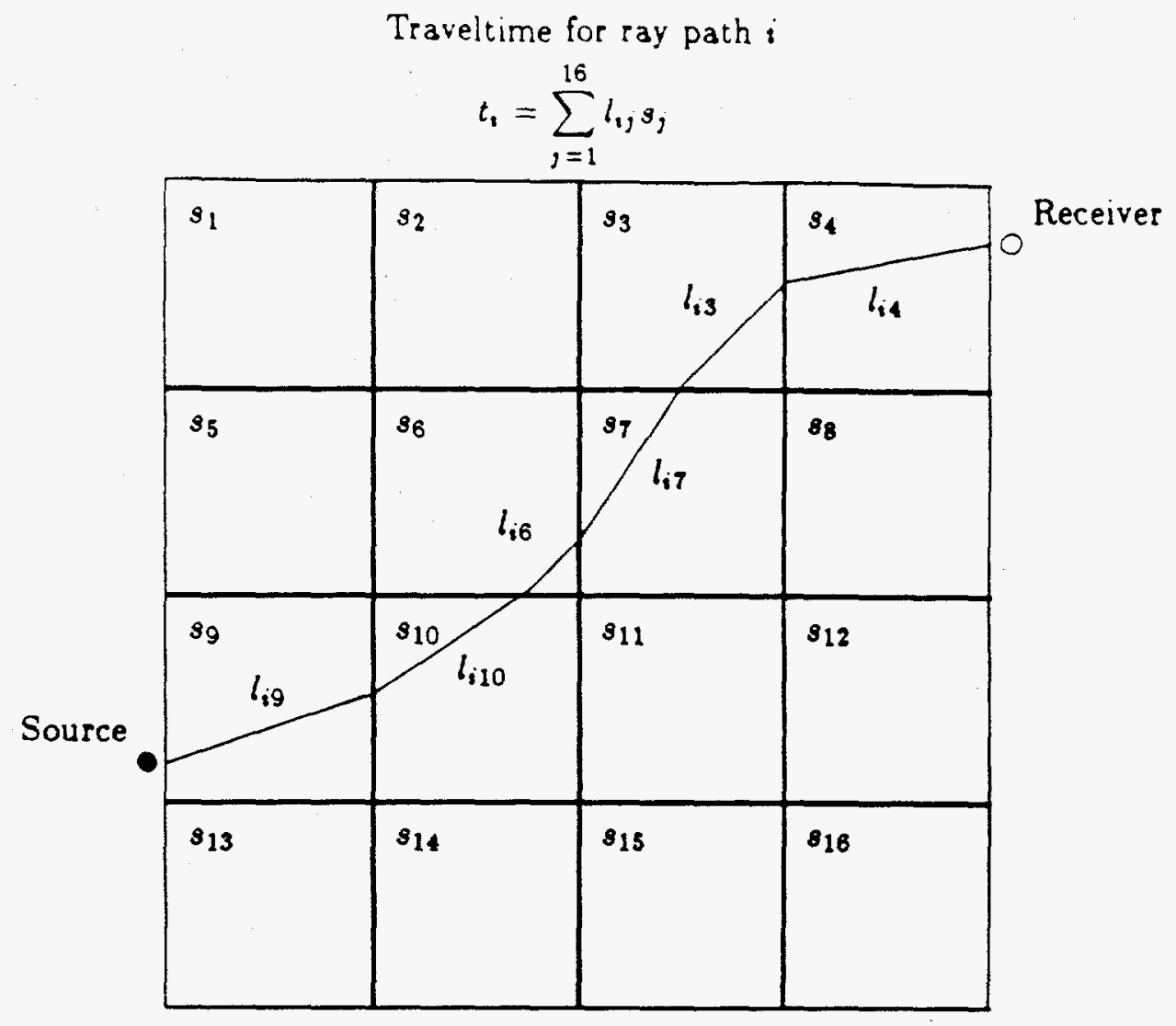

Figure 4: Schematic illustration of ray paths through a cell slownese model.

We can rewrite (24) in matrix notation by defining the column vectors $s$ and $t$ and the matrix $M$ as follows:

$$
=\left(\begin{array}{c}
s_{1} \\
s_{2} \\
\vdots \\
z_{n}
\end{array}\right), \quad \mathrm{t}=\left(\begin{array}{c}
t_{1} \\
t_{2} \\
\vdots \\
t_{m}
\end{array}\right), \quad \mathrm{M}=\left(\begin{array}{cccc}
l_{11} & l_{12} & \cdots & l_{1 n} \\
l_{21} & l_{22} & \cdots & l_{2 n} \\
\vdots & \vdots & \ddots & \vdots \\
l_{m 1} & l_{m 2} & \cdots & l_{m n}
\end{array}\right) .
$$

Equation (24) then becomes the basic equation of forward modeling for ray equation analysis:

$$
M_{s}=t .
$$

Note that equation (26) may be viewed as a numerical approximation to equation (21), i.e., it is just a discretized (or finite element) version of the equation. Equation (26) may be used for any set of ray paths, whether those ray paths minimize (21) or not. If the ray paths used to 
form the matrix $\mathbf{M}$ are actually minimizing ray paths, $\mathbf{M}$ is then implicitly a function of the slowness model $\mathbf{8}$.

The methods developed apply to both two-dimensional and three-dimensional imaging applications.

\subsection{Feasibility analysis for traveltime inversion}

The idea of using feasibility constraints in nonlinear programming problems is well established Fiacco and McCormick, 1990]. However, comparatively little attention has been given to the fact that physical principles such as Fermat's principle actually lead to rigorous feasibility constraints for nonlinear inversion problems [Berryman, 1991]. The main practical difference between the standard analysis in nonlinear programming and the analysis being developed for nonlinear inversion is that, whereas the functions involved in nonlinear programming are often continuous, differentiable, and relatively easy to compute explicitly, the functionals in nonlinear inversion (e.g., the traveltime functional) need not be continuous or differentiable and, furthermore, are very often rather difficult to compute. Feasibility constraints for such inversion problems are generally implicit, rather than explicit.

Equation (22) assumes that $P_{i}$ is one of Fermat's (least-time) paths and leads to the equalities summarized in the vector-matrix equation $\mathrm{Ms}_{s}=t$. Now suppose instead that $P_{i}$ is a trial ray path which may or may not be a least-time path. Fermat's principle allows us to write

$$
\int_{P_{i}} s(\mathbf{x}) d l^{P_{i}} \geq t_{i}
$$

where now $t_{i}$ is the measured traveltime for source-receiver pair $i$. When we discretize (27) for cell or block models and all ray paths $i$, the resulting set of $m$ inequalities may be written as

$$
\mathbf{M s} \geq \mathbf{t}
$$

Equations (27) and (28) can be interpreted as a set of inequality constraints on the slowneas model 8 . When 8 obeys these $m$ constraints, we say that 8 is feasible. When any of the constraints is violated, we say is infeasible. The set of inequalities collectively are called the feasibility constraints.

The concept of the feasibility constraint is quite straightforward in nonlinear programming problems [Fiacco and McCormick, 1990] whenever the constraints may be explicitly stated for the solution vector. However, in our inversion problems, an additional computation is required. The feasibility constraints are explicit for the traveltime data vector, but they are only implicit (i.e., they must be computed) for the slowness vector. This added degree of complication is unavoidable in the inversion problem, but nevertheless it is also very easily handled computationally with only very minor modifications of the usual nonlinear inversion algorithms.

\subsection{Algorithms}

Stable reconstruction algorithms using feasibility constraints to solve the traveltime tomography problem have been diacusaed in detail elsewhere [Berryman, 1990]. The key idea is to use the fact that the solution, if one exists, must lie on the feasibility boundary and then to force the 


\section{Scaling $s$ to find boundary point}

Model s

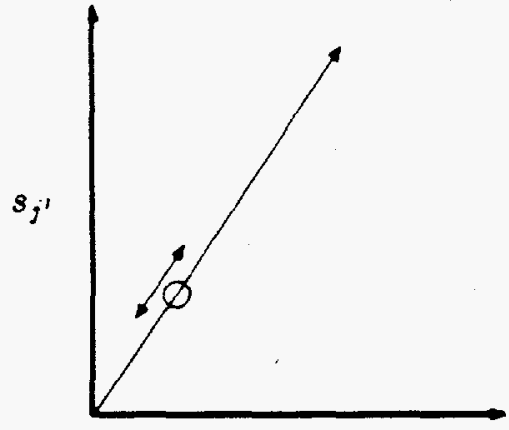

$s_{j}$
Data t

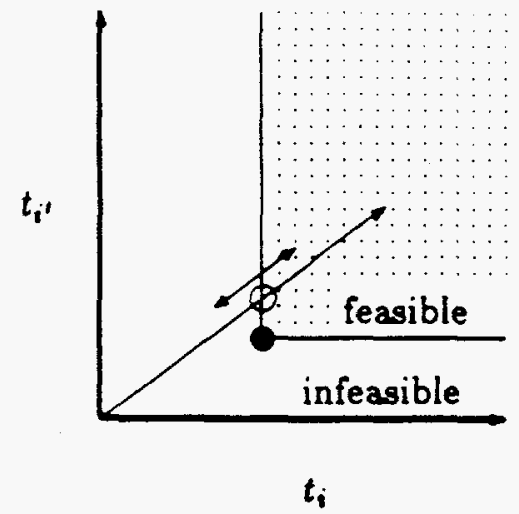

Figure 5: Scaling 8 to find feasibility boundary point. Feasibility boundary in data space is explicitly determined by the data. Feasibility boundary in model space is implicitly determined through the traveltime calculation.

Mapping the feasibility boundary

Model 8

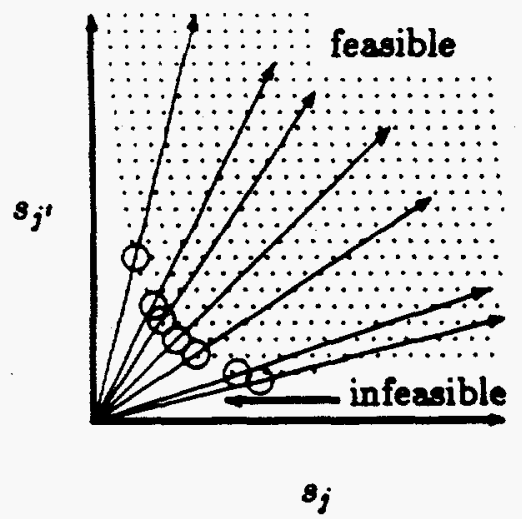

Data t

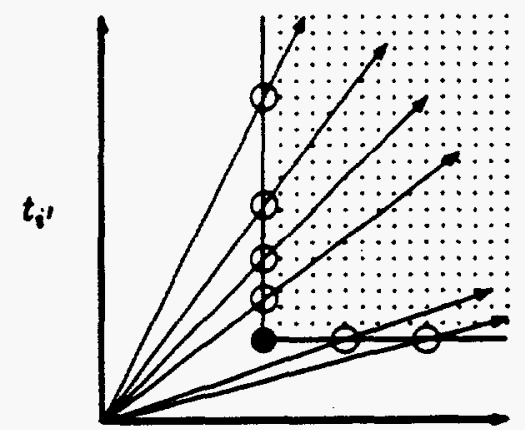

$t_{i}$

Figure 6: By scaling many slowneas vectors s, the location of the feasibility boundary in the model space can be mapped. 


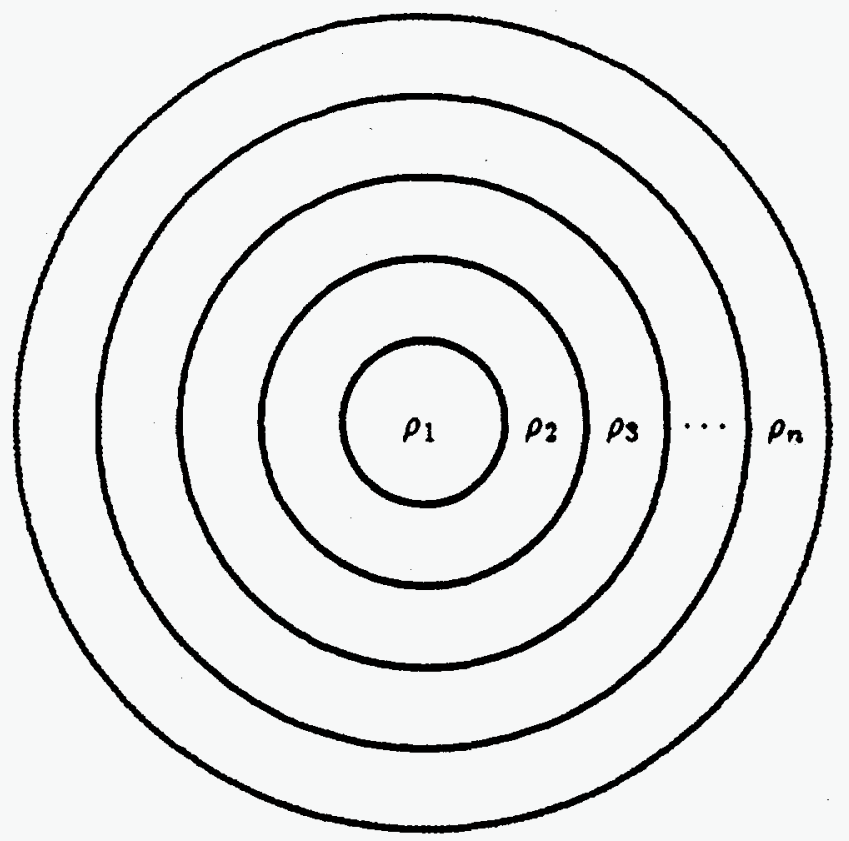

Figure 7: A sphere composed of concentric shells of constant density $\rho$ may be used to model free oscillations of the Earth. Compressional and shear wave velocities are assumed to have been previously determined using traveltime tomography. The inverse problem solves for density structure using measured eigenfrequencies of toroidal oscillation in this example.

solution in an iterative scheme to stay close to this manifold in the model space. An iterative linear least-squares inversion algorithm was easily and cheaply stablized using this approach. Other choices of algorithms based on the feasibility constraints are also possible, as will be discussed at the end of the paper.

\section{Feasibility constraints for density distribution in the Earth}

A somewhat different clase of problems involves analysis of free oecillations of the Earth in order to deduce its density structure [MacDonald and Ness, 1961; Gilbert, 1971; Jordan and Anderson, 1974; Aki and Richards, 1980; Gilbert, 1980; Ben-Menahem and Singh, 1981; Lapwood and Usami, 1981; Morelli and Driewonski, 1987; Snieder, 1993]. We concentrate on toroidal modes since they are independent of gravitational effects.

For each normal mode of a vibrating elastic medium, the potential energy density is composed of a bulk contribution $\kappa B$ proportional to the bulk modulus $\kappa$ and a shear contribution $\mu S$ proportional to the shear modulus. $B$ and $S$ are functionals of the exciting eigenfunctions. If the compressional and shear wave speeds ( $v_{c}$ and $v_{s}$, respectively) have been found using (for 
example) traveltime tomography, then we can use the identities

$$
e_{c}^{2}=(\kappa+4 \mu / 3) / \rho
$$

and

$$
v_{1}^{2}=\mu / \rho
$$

with $\rho$ being the mass density, to show that the potential energy density may be written in the form

$$
\rho U=\rho\left[\left(v_{c}^{2}-4 v_{c}^{2} / 3\right) B+v_{s}^{2} S\right]
$$

This equation shows explicitly that the potential energy is the sum of terms proportional to the unknown density, the squares of the (assumed) known velocities, and the functionals $B$ and $S$ of the (possibly trial) eigenfunctions.

The kinetic energy of a vibrating system is proportional to the density and the square of the time rate of change of the local displacement; so the Fourier transform of this energy density takes the general form $\omega^{2} \rho K$, where $\omega$ is the angular frequency of oscillation, and $K$ is the square of the trial displacement.

The partitioning of energy in a vibrating medium is known to balance the total kinetic and potential energy in the volume over a cycle. If the volume is $\Omega$, this balance between the two energies may be expressed in the equality

$$
\omega^{2} \int_{\Omega} \rho K d \Omega=\int_{\Omega} \rho U d \Omega
$$

where again $\rho$ is the local density, $K$ and $U$ are functionals of the eigenfunctions of the normal modes of vibration, and $\omega$ is the angular frequency of vibration for a particular mode. It is well-known that equation (32) can be rewritten in the form of a variational inequality as

$$
\omega^{2} \leq \frac{\int \rho U d \Omega}{\int \rho K d \Omega}
$$

in which the right hand side is again the Rayleigh-Ritz quotient, and the inequality itself follows from the Rayleigh-Ritz variational principle. In (33), $K$ and $U$ are now functionals of trial eigenfunctions, and equality is obtained when the minimum of the Rayleigh-Ritz quo tient is achieved, which occurs in variational calculations as the difference between the trial eigenfunction and the true eigenfunction approaches zero.

When looking for constraints on the density distribution $\rho$, we first notice that for this problem the Rayleigh-Ritz quotient does not place any constraint on the scale of the density. Multiplying any particular mass distribution $\rho$ by a positive constant $\gamma$ produces no change in this ratio, since the constant may be moved outside the integrals in both the numerator and the denominator and then cancels. This is an important difference between the present problem and the ones discussed previously (string vibration and traveltime inveraion). The boundary of the feasible set is not found by scaling the density in this problem, and therefore the shape of the feasibility boundary is that of a cone in the model space. 


\section{Feasibility analysis for free oscillations of the Earth}

Density model $\rho$

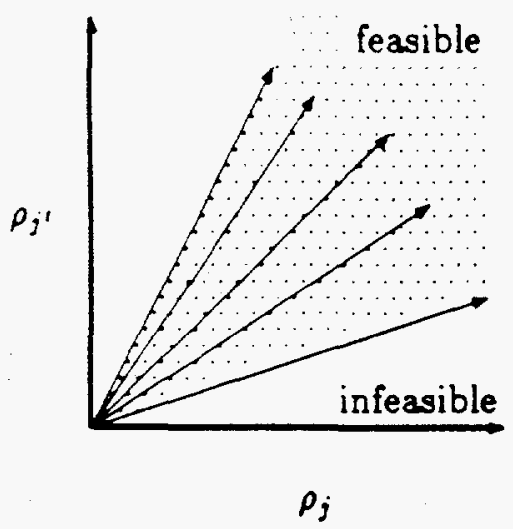

Frequency data $\omega$

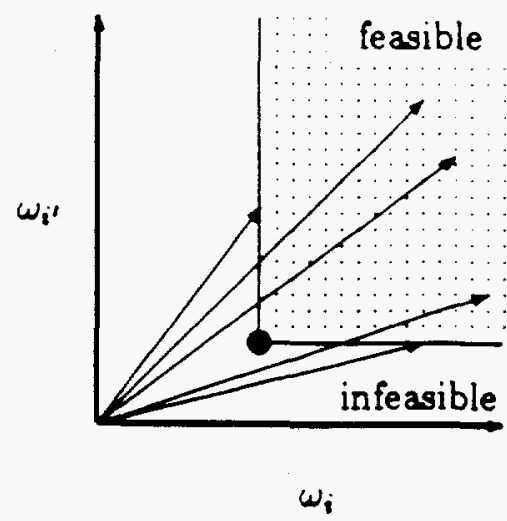

Figure 8: For free oscillations, scaling the density $\rho$ by a positive constant does not change either the trial eigenfunctions or the frequencies, showing that any point along a ray in density space is equally good for satisfying the frequency data. Thus, the feasible region forms a cone in the density space. of the Earth.

However, an independent constraint on the density scale is easily obtained when the total mass $M$ of a body such as the Earth is known, for then

$$
\int_{\Omega} \rho d n=M \text {. }
$$

Another constraint on the overall scale is provided by the moment of inertia

$$
\int_{\Omega} \rho r^{2} d \Omega=I
$$

when it is known.

To attack the inverse problem, we again introduce the concept of particular feasible density distributions and collections of these called "feasible sets," as before. We assume that some subset of the modal frequencies has been measured. For simplicity, we display equations for only one frequency and trial eigenfunction, but understand that the same argument applies simultaneously to all eigenfrequencies and trial eigenfunctions. Suppose that $p_{c}$ and $p_{b}$ are two density distributions, both satisfying the Rayleigh-Ritz inequality for the same trial eigenfunction. Thus, we are considering only local or trial feasibility of the densities in this first example. Then, by assumption,

$$
\omega_{n}^{2} \int \rho_{a} K d \Omega \leq \int \rho_{a} U d \Omega
$$

and

$$
\omega_{n}^{2} \int \rho_{b} K d n \leq \int \rho_{b} U d n
$$




\section{Constraints to fix the scale of the density}

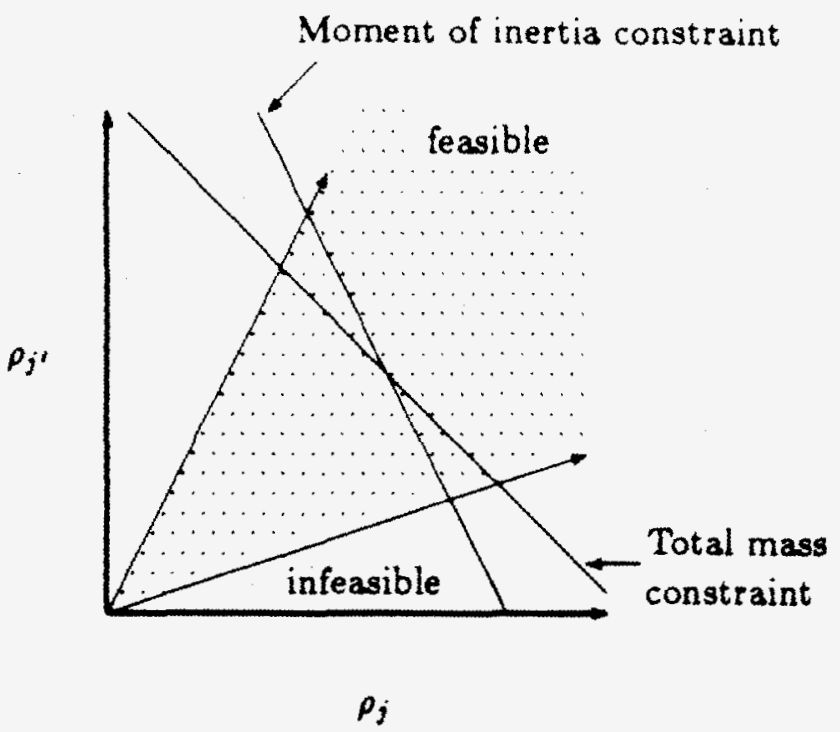

Figure 9: The scale of the density is determined by the total mass and/or the moment of inerits of the Earth.

both hold. If we next multiply (36) by $\lambda$ and $(37)$ by $(1-\lambda)$ and add the two inequalities, we find

$$
\omega_{n}^{2} \int \rho_{\lambda} K d \Omega \leq \int \rho_{\lambda} U d \Omega,
$$

where $\rho_{\lambda}=\lambda \rho_{a}+(1-\lambda) \rho_{b}$ is defined as the convex combination of the two densities. Thus, by the preceding argument, we have established the feasibility of the convex combination of any two density distributions that are themselves feasible (i.e., $p_{\lambda}$ also satisfies the RayleighRitz constraints obtained from the data) for any particular choice of trial eigenfunction. This argument establishes that local feasibility sets are convex.

The proof is similar for global feasibility. Suppoce we have found the actual eigenfunctions for the densities $\rho_{a}$ and $\rho_{b}$, and therefore know the corresponding potential and kinetic energy densities $K_{a}, U_{a}, K_{b}, U_{b}$ for these density distributions. These eigenfunctions (by assumption) produce minima in the respective Rayleigh-Ritz quotients; so if the resulting inequalities show that both $\rho_{a}$ and $\rho_{b}$ are feasible, then

$$
\omega_{n}^{2} \leq \frac{\int \rho_{a} U_{a} d \Omega}{\int \rho_{a} K_{a} d \Omega} \leq \frac{\int \rho_{a} U_{a} d \Omega}{\int \rho_{a} K_{a} d \Omega}
$$

and similarly

$$
\omega_{n}^{2} \leq \frac{\int \rho_{b} U_{b} d \Omega}{\int \rho_{b} K_{b} d \Omega} \leq \frac{\int \rho_{b} U_{*} d \Omega}{\int \rho_{b} K_{*} d \Omega}
$$


where the functionals $K$. and $U$. have been evaluated using some other convenient trial eigenfunctions (a specific choice will be made later in the discussion). Then, using the same argument as in the last paragraph, we find that the convex combination of densities $\rho_{\lambda}$ must satisfy

$$
\omega_{n}^{2} \leq \frac{\int \rho_{\lambda} U \cdot d \Omega}{\int \rho_{\lambda} K \cdot d \Omega}
$$

for any suitably constrained choice of trial eigenfunction. So, in particular the inequality must hold for the actual eigenfunctions that minimize the Rayleigh-Ritz quotient for (41), i.e., when $U_{*}=U_{\lambda}$ and $K_{*}=K_{\lambda}$. Thus, it follows from (41) that if $\rho_{a}$ and $\rho_{b}$ are globally feasible densities, then so is their convex combination $\rho_{\lambda}$ since

$$
\omega_{n}^{2} \leq \frac{\int \rho_{\lambda} U_{\lambda} d \Omega}{\int \rho_{\lambda} K_{\lambda} d \Omega}
$$

This proves that the global feasible set is convex. It is not hard to show that the global feasibility set may also be characterized as the intersection of all local feasibility sets, and is therefore a smaller (but still expected to be nonempty) set than any of the local feasibility sets (for particular trial eigenfunctions). It is harder to prove that the set is nonempty for this problem, than for the previous expamples.

\section{Simultaneous feasibility constraints for elastic moduli and density}

Now suppose that the only data available for determining Earth structure is the free oecillation data. What can be said about the corresponding nonlinear inversion problem? It turns out that the analysis goes through essentially as before, but now the model space is larger, involving not only the density distribution $\rho$ but also the bulk modulus $\alpha$ and the shear modulus $\mu$.

The Rayleigh-Ritz inequality is still of the form

$$
\omega^{2} \leq \frac{\int(\kappa B+\mu S) d \Omega}{\int \rho K d \Omega}
$$

When two models $\left(\rho_{a}, \kappa_{a}, \mu_{a}\right)$ and $\left(\rho_{b}, \kappa_{b}, \mu_{b}\right)$ satisfy the feasibility constraints, we have

$$
\omega_{n}^{2} \int \rho_{a} K d \Omega \leq \int\left(\kappa_{a} B+\mu_{a} S\right) d n
$$

and

$$
\omega_{n}^{2} \int \rho_{b} K d \Omega \leq \int\left(\kappa_{b} B+\mu_{b} S\right) d \Omega
$$

And the convex combination

$$
\left(\rho_{\lambda}, \kappa_{\lambda}, \mu_{\lambda}\right) \equiv\left(\lambda \rho_{a}+(1-\lambda) p_{b}, \lambda \kappa_{a}+(1-\lambda) \kappa_{b}, \lambda \mu_{a}+(1-\lambda) \mu_{b}\right)
$$

clearly satisfies the corresponding condition

$$
\omega_{n}^{2} \int \rho_{\lambda} K d \leq \int\left(\kappa_{\lambda} B+\mu_{\lambda} S\right) d \Omega
$$




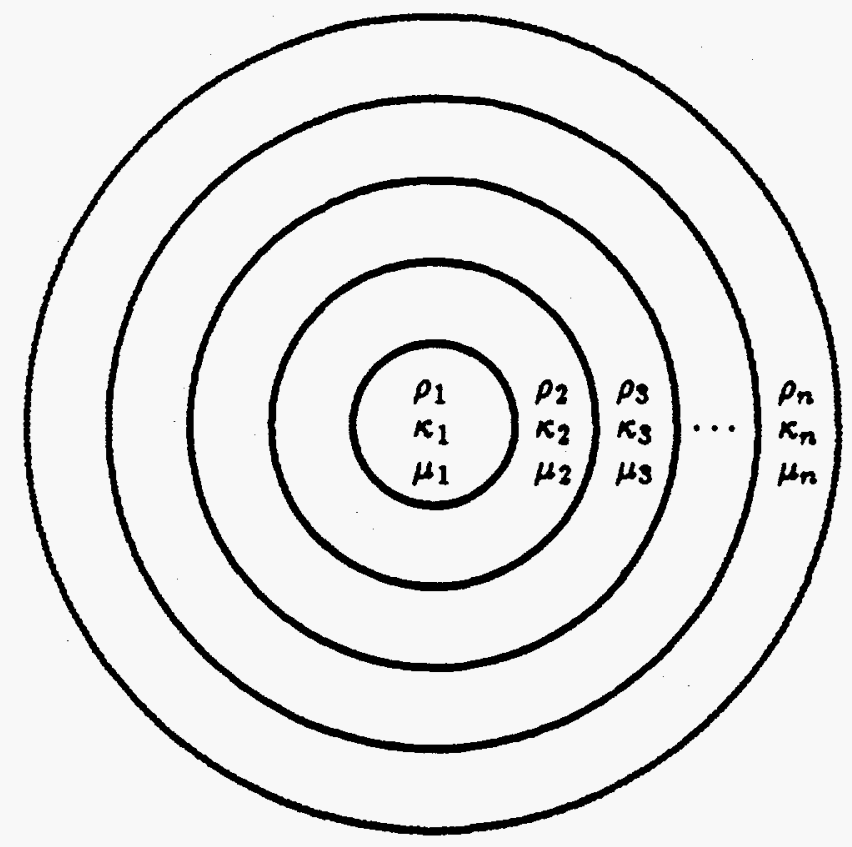

Figure 10: If compressional and shear velocities are not known, then a sphere composed of concentric shells of constant density $\rho$, bulk modulus $\kappa$, and shear modulus $\mu$ may be used to model free cocillations of the Earth. As in the last example, model structure is inverted from eigenfrequency data. 


\section{Free oscillations of the Earth without traveltime tomography}

Model space $(\rho, \kappa, \mu)$

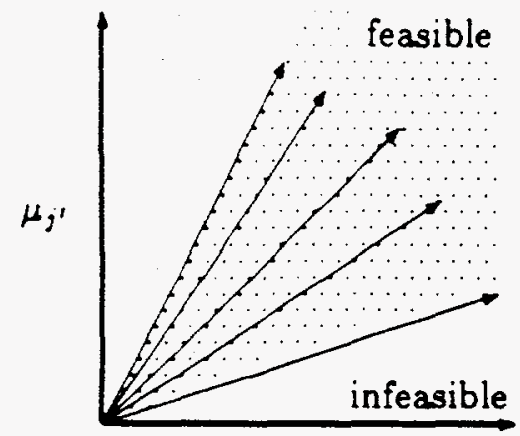

$\kappa_{j}$
Frequency data $\omega$

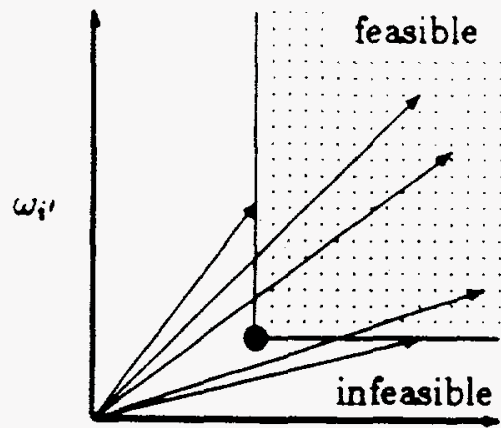

$\omega_{i}$

Figure 11: Scaling the triple $(\rho, \kappa, \mu)$ by a positive constant does not change either the trial eigenfunctions or the frequencies, showing again that any point along a ray in the model space is equally good for satisfying the frequency data. The overall scale is again determined by the total mass and/or the moment of inertia of the Earth.

where the same trial eigenfunctions have been used in (44)-(47). This again establishes convexity of any local feasibility set and the proof of the convexity of the global feasibility set for $(\rho, \kappa, \mu)$ follows immediately using the same arguments as before.

The Rayleigh-Ritz ratio is again scale invariant, so an overall change in scale of the form $(\rho, \kappa, \mu) \rightarrow(\gamma \rho, \gamma \kappa, \gamma \mu)$ does not affect the frequency predictions. But, as long as the scale of any one of the parameters is known, the others follow from it, so knowledge of the total mass (34) and/or total moment of inertia (35) is again sufficient to determine the scale of the model.

The main difference between the results for a single parameter $\rho$ and those for a set of parameters such as the triple $(\rho, \kappa, \mu)$ is that, depending on details of how the three parameters are discretized for numerical treatment, the model space will be on the order of three times larger for the case of pure free cacillation data and therefore correspondingly more of this vibration data will be required to obtain enough constraints to produce the same degree of model resolution.

\section{Variational structure for inverse problems}

The various inverse problems considered so far may be viewed as special cased of the following general problem.

Suppose we have the set of measured data $\left\{q_{i}\right\}$ and that these data are known - from the mathematical physics of the related forward problem - to be minima of an appropriate 
variational functional so that

$$
q_{i} \leq Q_{1} n_{1}, \ldots, n_{k} ; d_{1}, \ldots, d_{m} ;
$$

where $Q_{2}$ is a functional whose $(k+m)$ arguments are parameters of the model (such as density for string vibrations, slowness in traveltime tomography, or bulk and shear moduli in elastic vibration). There are additional dependencies on the trial eigenfuctions contained implicitly in (48), but we suppress these in the present notation. If the functional can be written in the form of a quotient

$$
Q_{i}\left[n_{1}, \ldots, n_{k} ; d_{1}, \ldots, d_{m}\right]=\frac{N_{i}\left[n_{1}, \ldots, n_{k}\right]}{D_{i}\left[d_{1}, \ldots, d_{m}\right]},
$$

where the functionals in the numerator $N_{i}$ and in the denominator $D_{i}$ are the nselves linear functionals of each of their arguments, then we find that all the examples considered may be written in this form (see Table 1).

It follows easily from this postulated form that the inverse problem leads in all cases to a convex feasible region in the multiparameter model space. This follows directly from the observation that, if

$$
q_{i} D_{i}\left[\hat{d}_{1}, \ldots, \hat{d}_{m}\right] \leq N_{i}\left[\hat{n}_{1}, \ldots, \hat{n}_{k}\right] \text { for all } i
$$

and if

$$
q_{i} D_{i}\left[\tilde{d}_{1}, \ldots, \tilde{d}_{m}\right] \leq N_{i}\left[\tilde{n}_{1}, \ldots, \tilde{n}_{k}\right] \text { for all } i
$$

then for any $\lambda$ in the range $0 \leq \lambda \leq 1$ we find

$$
q_{i} \leq \frac{N_{i}\left[n_{1}^{(\lambda)}, \ldots, n_{k}^{(\lambda)}\right]}{D_{i}\left[d_{(\lambda)}, \ldots, d_{m}^{(\lambda)}\right]} \text { for all } i
$$

where

$$
n_{j}^{(\lambda)}=\lambda \hat{n}_{j}+(1-\lambda) \tilde{n}_{j} \quad \text { for } \quad 1 \leq j \leq k
$$

and

$$
d_{l}^{(\lambda)}=\lambda \hat{d}_{l}+(1-\lambda) \tilde{d}_{i} \text { for } 1 \leq l \leq m
$$

guaranteeing that the convex combinations of the "hat" and "tilde" models parametrized by $\lambda$ are also feasible if the "hat" and "tilde" models are themselves feasible. The arguments given previously may be repeated to demonstrate the existence of a convex feasible set for all such problems.

TABle 1. 


\begin{tabular}{|l|c|c|c|l|}
\hline Problem & $\left\{q_{1}\right\}$ & $\left\{n_{1}\right\}$ & $\left\{d_{1}\right\}$ & Description of feasible set \\
\hline $\begin{array}{l}\text { Density from } \\
\text { string vibration }\end{array}$ & $\left\{\omega_{i}\right\}$ & - & $d_{1}=\rho$ & $\begin{array}{l}\text { Finite, bounded region } \\
\text { near the origin }\end{array}$ \\
$\begin{array}{l}\text { Wave slowness from } \\
\text { traveltime tomography }\end{array}$ & $\left\{r_{1}\right\}$ & $n_{1}=s$ & - & $\begin{array}{l}\text { Infinite, bounded away } \\
\text { from the origin }\end{array}$ \\
Elastic constants from & $\left\{\omega_{i}\right\}$ & $n_{1}=\kappa$ & $d_{1}=\rho$ & Infinite cone \\
free oscillations & $n_{2}=\mu$ & & \\
\hline
\end{tabular}

\section{Discussion}

\subsection{Finite data sets}

Suppose we have a finite set of measurements $\left\{q_{1}, \ldots, q_{M}\right\}$ and we compute the feasible set $\mathcal{F}_{M}$ associated with that measurement set. Now suppose that an additional measurement is made so the measurement set is now $\left\{q_{1}, \ldots, q_{M+1}\right\}$ with the associated feasible set $\xi_{M+1}$. What is the relationship between $\bar{F}_{M}$ and $\xi_{M+1}$ ? The addition of a constraint can only decrease the size of the feasible set, so it follows easily from the convexity properties that in general $\xi_{M+1} \subseteq \xi_{M} \subseteq \xi_{M-1} \subseteq \cdots \subseteq \xi_{1}$.

\subsection{Measurement error}

An issue that is often raised about the usefulness of the feasibility constraints concerns the effects of measurement errors on the location of the feasibility boundary. The variational constraint equations always find the dats in the role of upper or lower bounds on integrals involving the unknown parameters. The data therefore enters these constraints linearly, so small measurement errors will generally lead to small (or possibly no) changes in the location of the bounding curves, depending on which measurements are in error and which measurements are the most constraining. We can think of the feasibility boundary in these circumstances, not as a sharp but rather, as a fuzzy boundary. If the errors are small, then the fuzzy region is also small. It is useful to take this fuzziness into account in practical algorithms that make use of the feasibility boundary to reconstruct the desired model parameters in our inverse problems. This can be accomplished by using either the least (or the most) constraining range of data-minuserror (or data-plus-error) when computing our estimate of the boundary location. Alternatively, the fuzziness of the boundary can be incorporated directly into algorithms that use only the approximate location of the feasibility boundary as a means of constraining a search for the desired model parameters based on other criteria (such as minimining the least-equares error in predicted versus measured data, or some other choices of objective functional minimization). Experience has shown that practical algorithms based on feasibility constraints are leas sensitive (more robust) to the presence of measurement errors than most other algorithms for inversion. 


\subsection{Algorithms}

The variational structure of nonlinear inverse problems does not by itself provide an algorithm for reconstructing the desired model parameters from the data. Nevertheless, knowing the existence of the feasibility boundary and the convex nature of the feasible set suggests a number of possible algorithms that may be useful depending on the particular problem. We may suggest three types of algorithms: (1) The most obvious and probably the least practical algorithm entails a search along the feasibility boundary for the model that best fits the data. This approach has much in common with linear programming methods of the simplex type, but is probably not practical for most of the large dimensional problems that would benefit from the methods discussed in this paper. The reason for the difference is that the feasibility boundary in nonlinear inversion problems is determined only implicitly and therefore requires considerable computation to find each boundary point, whereas in linear and most other nonlinear programming problems the boundary constraints are given explicitly. (2) A Monte Carlo or shooting method that tries to sample a region of the model space and map the feasibility boundary in that local region has been tried on both the string density inversion problem and on the traveltime inversion problem. This approach has been found to work extremely well in the problems of lower dimensionality, and it also works well in higher dimensional problems that are easily parallelized (such as traveltime tomography) when many processors are available. Finally, (3) virtually any existing inversion algorithm can be easily modified to incorporate the feasibility constraints as a natural means of regularizing, i.e., preventing divergences from occurring. In particular, iterative linesr least-squares inversion algorithms that might diverge due to inconsistencies arising from the forward modeling (trial eigenfuctions or trial ray paths) based on a previous guess of the model can be easily stabilized by forcing the stepsize for model updates to remain small enough so that the successive iterates do not wander away from the feasibility boundary. Such a constraint does not tie the result to any particular part of the model space that must be chosen prior to the inversion (i.e., hard constraints on the maximum and minimum values of the parameters in the model are not needed), but rather tethers the final result to a manifold determined strictly by the data and the measurement configuration. Thus, the data itself is used to determine the appropriate means of regularizing the solution to the inverse problem in such algorithms. This approach is probably the one that will find the most use in practical solutions to inverse problems.

\section{ACKNOWLEDGMENTS}

The numerical experiments for the string vibration problem were performed by $O$. $S$. Tai, a summer research atudent visiting at LLNL. I also thank G. C. Berozs, J. M. Rice, and G. Zandt for helpful leads into the literature on free oscillations of the Earth. This work was performed under the auspices of the U. S. Department of Energy by the Lawrence Livermore National Laboratory under contract No. W-7405-ENG-48 and supported specifically by the Geosciences Research Program of the DOE Office of Energy Research within the Office of Basic Energy Sciences, Division of Engineering and Geosciences. Part of this work was done while the author was visiting the Geophysics Department at Stanford University. Partial support for this visit was provided by the Stanford Exploration Project, whose sponsors are hereby gratefuly acknowledged.

\section{REFERENCES}


K. Aki and P. G. Richards, Quantitative Seismology: Theory and Methods, Vol. I, Freeman, Vew York, 1980, Chapter 8, pp. 337-381.

A. Ben-Mena..m and S. J. Singh, Seismic Woves and Sources, Springer-Verlag, New York, 1981, Chapter 6, pp. 337-419.

J. G. Berryman, Stable iterative reconstruction algorithm for nonlinear traveltime tomography, Inverse Problems 6, 21-42 (1990).

J. G. Berryman, Convexity properties of inverse problems with variational constraints, $J$. Eranklin Inst. 328, 1-13 (1991).

E. A. Coddington and N. Levinson, Theory of Differential Equations, McGraw-Hill, New York, 1955, pp. 208-221.

R. Courant and D. Hilbert, Methods of Mathematical Physics, Vol. I, Interscience, New York, 1953, pp. 132-134.

K. A. Dines and R. J. Lytle, Computerized geophysical tomography, Proc. IEEE 67,1065-1073 (1979).

A. V. Fiacco and G. P. McCormick, Nonlinear Programming: Sequential Unconstrained Minimization Techniques, SIAM, Philadelphia, 1990, Chapter 6, 86-112.

F. Gilbert, Inverse problems for the Earth's normal modes, in Mathematical Problems in the Geophysical Sciences, 2, Lectures in Applied Mathematics, vol. 14, edited by W. H. Reid (American Mathematical Society, 1971), pp. 107-121

F. Gilbert, An introduction to low-frequency seismology, in Proceedings of the International School of Physics "Enrico Fermi", Course LXXVIII, edited by A. M. Driewonski and E. Boschi, North-Holland, Amsterdam, 1980, pp. 41-81.

T. H. Jordan and D. L. Anderson, Earth structure from free oscillations and travel times, Geophys. J. R. A str. Soc. 36, 411-459 (1974).

E. R. Lapwood and T. Usami, Free Oscillations of the Earth, Cambridge University Press, London, 1981.

R. J. Lytle and K. A. Dines, Iterative ray tracing between boreholes for underground image reconstruction, IEEE Trans. Geosci. Remote Sers. 18, 234-240 (1980).

G. J. F. MacDonald and N. Ness, A study of the free oscillations of the earth, J. Geophys. Ree. 66, 1865-1911 (1961).

A. Morelli and A. M. Dziewonski, The harmonic expansion approach to the retrieval of deep Earth structure, in Seismic Tomography: With Applications in Global Seiomology and Exploration Geophysics, edited by G. Nolet, Reidel, Dordrecht, 1987, Chapter 11, pp. 251-274.

R. Snieder, Global inversions using normal modes and long-period surface waves, in Seiomic Tomography: Theory and Practice, edited by H. M. Iyer and K. Hirahara, Chapman and Hall, London, 1993, Chapter 3, pp. 23-63. 